\title{
Survey over VANET Routing Protocols for Vehicle to Vehicle Communication
}

\author{
Bijan Paul, ${ }^{1}$ Mohammed J. Islam ${ }^{2}$ \\ 1,2Department of Computer Science \& Engineering Shahjalal University of Science \& Technology, Sylhet, \\ Bangladesh
}

\begin{abstract}
VANET (Vehicular Ad-hoc Network) is an emerging new technology with some unique characteristics that makes it different from other ad hoc network. Due to rapid topology changing and frequent disconnection it is also difficult to design an efficient routing protocol for routing data among vehicles, called $V 2 \mathrm{~V}$ or vehicle to vehicle communication and vehicle to road side infrastructure, called V2I. Because of road accident daily occurrence VANET is one of the influencing areas for the improvement of Intelligent Transportation System (ITS) which can increase road safety and provide traffic information etc. The existing routing protocols for VANET are not efficient to meet every traffic scenarios. Suitable routing protocols are required to establish communication between vehicles in future for road safety. In this paper, we focus on the merits and demerits of routing protocols which will help to develop new routing protocols or improvement of existing routing protocol in near future.
\end{abstract}

Keywords: Proactive, Reactive, Routing Protocol, VANET, MANET

\section{Introduction}

VANET is a new technology in recent years; researchers have taken huge attention on it. There are many research projects around the world which are related with VANET such as COMCAR [1], DRIVE [2], FleetNet [3] and NoW (Network on Wheels) [6], CarTALK 2000 [17], CarNet [18]. VANET is an autonomous $\&$ self organizing wireless communication network. In this network the cars are called nodes which involve themselves as servers and/or clients for exchanging \& sharing information. There are number of applications are envisioned for VANET, some of which are already exist in some recently designed vehicles and is shown in figure 1.

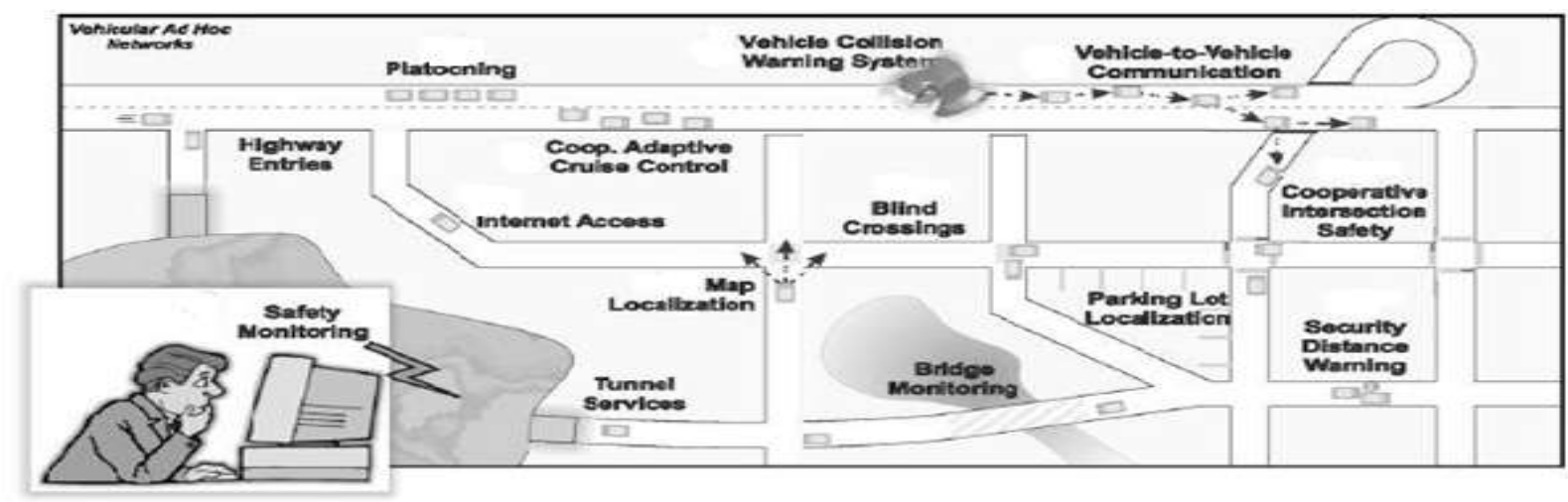

Fig.1 VANET Applications

In modern age, by using VANETs vehicles are capable to interact with themselves which help to avoid any critical situation such as road side accidents, traffic jams, speed control, free passage of emergency vehicles and unseen obstacles etc.

VANET has some unique characteristics which make it different from MANET as well as challenging for designing VANET applications.

There are several papers related to performance evaluation of VANET routing protocols. But due to the movement behavior of vehicles that are quite different from the original ad hoc network system, designing a new routing protocol in VANET is very challenging. In this research paper we focus on VANET routing protocols and their merits and demerits. The remainder of the paper is organized as follows: Section II describes overview of routing protocol in VANET and section III, IV, V, VI, VII discusses merits and demerits of Topology based Routing Protocols, Position Based Routing Protocols, Cluster Based Protocols, Broadcast Protocols, and Geocast Protocols. We conclude in section VIII and at the end add references. 


\section{Overview Of Routing Protocols}

The characteristic of highly dynamic topology makes the design of efficient routing protocols for VANET challenging. The routing protocols of VANET can be classified into five categories such as Topology based Routing Protocol, Position based Routing Protocol, Cluster based Routing Protocol, Geocast based Routing Protocol, and Broadcast based Routing Protocol. It is shown is table 1.

Table 1: Overview of routing protocols

\begin{tabular}{|l|l|l|l|l|l|}
\hline $\begin{array}{l}\text { Type of } \\
\text { Protocols }\end{array}$ & Topology Based & Position Based & Cluster Based & $\begin{array}{l}\text { Geocast } \\
\text { Based }\end{array}$ & $\begin{array}{l}\text { Broadcast } \\
\text { Based }\end{array}$ \\
\hline $\begin{array}{l}\text { Forwarding } \\
\text { Technique }\end{array}$ & $\begin{array}{l}\text { Wireless multi } \\
\text { hop Forwarding }\end{array}$ & $\begin{array}{l}\text { Heuristic } \\
\text { Technique }\end{array}$ & $\begin{array}{l}\text { Wireless multi } \\
\text { hop Forwarding }\end{array}$ & $\begin{array}{l}\text { Wireless multi } \\
\text { hop } \\
\text { Forwarding }\end{array}$ & $\begin{array}{l}\text { Wireless multi } \\
\text { hop } \\
\text { Forwarding }\end{array}$ \\
\hline $\begin{array}{l}\text { Strategy of } \\
\text { Recovery }\end{array}$ & $\begin{array}{l}\text { Multi Hop } \\
\text { Forwarding }\end{array}$ & $\begin{array}{l}\text { Carry } \\
\text { Forward } \\
\text { approach }\end{array}$ & $\begin{array}{l}\text { Carry } \\
\text { Forward } \\
\text { approach }\end{array}$ & Flooding & $\begin{array}{l}\text { Carry } \\
\text { Forward } \\
\text { approach }\end{array}$ \\
\hline $\begin{array}{l}\text { Digital Map } \\
\text { Requirement }\end{array}$ & No & Yes & No & No \\
\hline $\begin{array}{l}\text { Virtual } \\
\text { Infrastructure } \\
\text { Requirement }\end{array}$ & No & No & Yes & No & No \\
\hline $\begin{array}{l}\text { Realistic } \\
\text { Traffic Flow }\end{array}$ & Yes & Yes & No & Yes & Yes \\
\hline Scenario & Urban & Urban & Urban & Highway & Highway \\
\hline
\end{tabular}

\section{Topology Based Routing Protocol}

Topology based routing protocols use link's information within the network to send the data packets from source to destination. Topology based routing approach can be further categorized into proactive (tabledriven) and reactive (on-demand) routing.

\subsection{Proactive (table-driven)}

Proactive routing protocols are mostly based on shortest path algorithms. They keep information of all connected nodes in form of tables because these protocols are table based. Furthermore, these tables are also shared with their neighbors. Whenever any change occurs in network topology, every node updates its routing table.

Pros

-No route discovery is required.

-Low latency for real time applications.

Cons

-Unused paths occupy a significant part of the available bandwidth.

\subsubsection{Fisheye State Routing}

FSR [19] is a proactive or table driven routing protocol where the information of every node collects from the neighboring nodes. Then calculate the routing table. It is based on the link state routing \& an improvement of Global State Routing.

Pros

-FSR reduces significantly the consumed bandwidth as it exchanges partial routing update information with neighbors only.

-Reduce routing overhead.

-Changing in the routing table will not occur even if there is any link failure because it doesn't trigger any control message for link failure.

Cons

-Very poor performance in small ad hoc networks.

-Less knowledge about distant nodes.

-The increase in network size the storage complexity and the processing overhead of routing table also increase. -Insufficient information for route establishing.

\subsection{Reactive (On Demand)}

Reactive routing protocol is called on demand routing because it starts route discovery when a node needs to communicate with another node thus it reduces network traffic. 
Pros

-To update routing table not require periodic flooding the network. Flooding requires when it is demanded.

-Beaconless so it saves the bandwidth.

Cons

-For route finding latency is high.

-Excessive flooding of the network causes disruption of nodes communication.

\subsubsection{Ad Hoc on Demand Distance Vector (AODV)}

AODV routing protocol [9] is a reactive routing protocol which establish a route when a node requires sending data packets. It has the ability of unicast $\&$ multicast routing. It uses a destination sequence number (DestSeqNum) which makes it different from other on demand routing protocols.

Pros

-An up-to-date path to the destination because of using destination sequence number.

-It reduces excessive memory requirements and the route redundancy.

-AODV responses to the link failure in the network.

-It can be applied to large scale adhoc network.

Cons

-More time is needed for connection setup \& initial communication to establish a route compared to other approaches.

-If intermediate nodes contain old entries it can lead inconsistency in the route.

-For a single route reply packet if there has multiple route reply packets this will lead to heavy control overhead. -Because of periodic beaconing it consume extra bandwidth.

\subsubsection{Dynamic Source Routing}

The Dynamic Source Routing (DSR) [10] protocol presented in which utilize source routing \& maintain active routes. It has two phases route discovery \& route maintenance.

\section{Pros}

-Beacon less.

-To obtain route between nodes, it has small overload on the network. It uses caching which reduce load on the network for future route discovery.

-No periodical update is required in DSR.

Cons

-If there are too many nodes in the network the route information within the header will lead to byte overhead.

-Unnecessary flooding burden the network.

-In high mobility pattern it performs worse.

-Unable to repair broken links locally.

\subsubsection{Temporally Ordered Routing Protocol (TORA)}

Temporally Ordered Routing Protocol [11] is based on the link reversal algorithm that creates a direct acyclic graph towards the destination where source node acts as a root of the tree. In TORA packet is broadcasted by sending node, by receiving the packet neighbor nodes rebroadcast the packet based on the DAG if it is the sending node's downward link.

Pros

-It creates DAG (Direct acyclic graph) when necessary.

-Reduce network overhead because all intermediate nodes don't need to rebroadcast the message.

-Perform well in dense network.

Cons

-It is not used because DSR \& AODV perform well than TORA.

-It is not scalable.

\section{Position Based Routing Protocol}

In position based routing, each node knows it's own and neighbor node geographic position by position determining services like GPS. It does not maintain any routing table or exchange any link state information with neighbor nodes. Information from GPS device is used for routing decision. Various types of position based greedy V2V protocols are GSR, GPSR, GPCR, CAR, A-STAR, STBR etc.

Pros

-Route discovery \& management is not required.

-Scalability.

-Suitable for high node mobility pattern. 
Cons

-It requires position determining services.

-GPS device doesn't work in tunnel because satellite signal is absent there.

\subsection{Greedy Perimeter Stateless Routing (GPSR)}

Greedy Perimeter Stateless Routing [12] selects a node which is closest to the final destination by using beacon. It uses greedy forwarding algorithm if it fails it uses perimeter forwarding for selecting a node through which a packet will travel.

Pros

-To forward the packet a node needs to remember only one hop neighbor location.

-Forwarding packet decisions are made dynamically.

Cons

-For high mobility characteristics of node, stale information of neighbors' position is often contained in the sending nodes' neighbor table.

-Though the destination node is moving its information in the packet header of intermediate node is never updated.

\subsection{Greedy Perimeter Coordinator Routing (GPCR)}

Greedy Perimeter Coordinator Routing [13] is a position-based routing protocol uses greedy algorithms to forward packet based on a pre-selected path which has been designed to deal with the challenges of city scenarios. No global or external information like static map does not require in GPCR.

Pros

-Does not require any global or external information.

-For representing the planar graph it uses the underlying roads though it is based on the GPSR.

-It has no as usual a planarization problem like unidirectional links, planar sub-graphs \& so on.

Cons

-Depends on junction nodes.

-There has a problem in the Junction detection approach in which first approach fails on curve road \& second approach fails on a sparse road.

\subsection{Connectivity-Aware Routing (CAR)}

For city and/or highway environment CAR [20] is designed which uses AODV for path discovery and uses PGB for data dissemination mode. It uses guard concept to maintain the path.

Pros

-No digital map is required.

-It has no local maximum problem.

-CAR ensures to find the shortest connected path because CAR has higher packet delivery ratio than GPSR and GPSR+AGF.

Cons

-Unnecessary nodes can be selected as an anchor.

-It cannot adjust with different sub-path when traffic environment changes.

\subsection{Geographic Source Routing (GSR)}

GSR [7] routing was proposed for vehicular ad hoc networks in city environments which is the combination of position-based routing with topological knowledge. GSR uses greedy forwarding along a preselected shortest path \& this path is calculated by using Dijkstra algorithm.

Pros

-Packet delivery ratio of GSR is better than AODV \& DSR.

-GSR is scalable than AODV \& DSR.

Cons

-This protocol neglects the situation like sparse network where there are not enough nodes for forwarding packets.

-GSR shows higher routing overhead than GyTAR because of using hello messages as control messages.

4.5 Anchor-Based Street and Traffic Aware Routing (A-STAR)

A-STAR [15] is a position based routing protocol which is specially design for city scenarios for inter vehicle communication system. It ensures high connectivity in packet delivery by using vehicular traffic city bus information for an end-to-end connection.

Pros

-In low traffic density, A-STAR ensures for finding an end-to-end connection. 
-By comparing with the greedy approach of GSR \& the perimeter mode of GPSR. A-STAR uses a new local recovery strategy which is more suitable for city environment.

-Path selection of A-STAR ensures high connectivity though its packet delivery ratio is lower than GSR \& GPSR.

Cons

-Packet delivery ratio of A-STAR is lower than GSR \& GPSR.

-To find a path from source to destination it uses static information based on city bus routes which causes connectivity problem on some portion of streets.

\subsection{Street Topology Based Routing (STBR)}

Street Topology-Based Routing (STBR) [4] is based on the idea of elucidate a given street map as a planar graph which has three valid states: master, slave, and forwarder for a node. In STBR one node is selected as a master on a junction, other nodes act as slaves \& intermediate nodes between junctions act as forwarders.

Pros

-It traverses least spanning multiple junctions for long distance unicast communication.

Cons

-STBR is not appropriate for mixed scenarios because it would try to send junction beacons along a highway.

-In STBR complexity increases because of some special cases like transferring the two-hop neighbor table to the new master when the old master leaves the junction.

\section{Cluster Based Routing Protocol}

Cluster based routing protocol makes cluster between nodes or vehicles. A group of nodes identifies themselves to be a part of cluster and each cluster has only one cluster-head, which is responsible for intra and inter-cluster communication. For intra-cluster communication within each cluster is connected via direct link and for inter-cluster communication is connected via cluster-head. In clustering, cluster head broadcast the packet to cluster which provides good scalability for large networks although network delay and overhead increase for high mobility feature of VANET. The various Clusters based routing protocols are HCB, CBDRP, CBLR, CBR etc.

\subsection{Hierarchical Cluster Based Routing (HCB)}

HCB [21] uses clustering technique which is designed for highly mobility adhoc networks. Two layers communication architecture is used in HCB. Layer-1 nodes have single radio interface and they are communicate with each other via multi-hop path. In layer-2 nodes are communicate with each other via base station.

Pros

-Intra-cluster routing is performed independently in each cluster.

-Cluster heads exchange membership information periodically to enable inter-cluster routing.

Cons -Number of retransmission is high because of high packet loss.

\subsection{Cluster-Based Directional Routing Protocol (CBDRP)}

CBDRP [22] is designed for vehicles which are moving in same direction. Source node sends packet to cluster header then it is the responsibility of the cluster header to forward the packet which is in the same cluster. This protocols behavior is like CBR but direction and velocity are consider when packet forwarding.

Pros

-Link stability problem solve in VANET.

-Reliable and rapid data transfer.

Cons

-Control packet overhead is average.

-Number of retransmission is high.

\subsection{Cluster Based Location Routing (CBLR)}

CBLR [16] is not only cluster based protocol but also a reactive or on demand routing protocol. A routing table is used by each cluster header which contains addresses and locations of the cluster members. Cluster header also track information about all neighboring clusters by using Cluster Neighbor Table. The packet transmission mechanism is that when a source node wants to send a packet to the destination then it sends the packet to the closest neighbor to the destination if it is in same cluster. If destination is in another cluster then stores the data packet in its buffer then broadcasts Location Request (LREQ) packets and starts a timer. 
Pros

-Suitable for high mobility network.

-Digital map is used

-Control packet overhead is low.

Cons -Number of retransmission is high.

\subsection{Cluster Based Routing (CBR)}

CBR [23] is a routing protocol which is based on position and cluster where the geographic area is divided into square grids. By using geographical information each node forward data to the next node. If there is a vehicle in a grid is elect as a cluster header then it broadcasts a LEAD message to its neighbors and whenever the header is leaving the grid, it will broadcast LEAVE message containing its grid position.

Pros -Need not discover route so routing overhead is less.

Cons -This protocol doesn't consider velocity and direction which is important parameter for VANET.

\subsection{Location Routing Algorithm with Cluster- Based Flooding(LORA-CBF)}

LORA-CBF [14] is same as greedy routing. The cluster-head is responsible for maintain information between nodes. When two clusters is connected by a node then it is called gateway. When destination of a node is not available then cluster head and gateways send location request (LREQ) packets.

Pros

-Packet forwarding same as greedy routing

Cons

-Heterogeneous performance results.

\section{Broadcast Based Routing Protocol}

Broadcast routing protocol is flooding based routing protocol which is used in VANET for sharing information among vehicles such as when an accident or an event occurs then transmit the information to maximum nodes possible. Broadcast send a packet to all nodes in the network which causes exponential increase in message transmission leading to collisions, higher bandwidth consumption and drop in overall performance. Although this protocol performs better when small numbers of nodes are involved. Various Broadcast routing protocols are BROADCOMM, UMB, V-TRADE, DV-CAST, EAEP, SRB, PBSM, PGB, DECA, POCA etc.

\subsection{BROADCOMM}

BROADCOMM [24] is based on hierarchal structure which is used for highway network. In this protocol highway is segmented to virtual cells which move along with the vehicles. There are two level of hierarchy for the nodes in the highway. All the nodes in a cell are included in first level. The second level is represented by cell reflectors (few nodes in each virtual cell) are responsible for handling messages within its cell nodes and forwarding or receiving the messages to or from neighboring cell reflectors.

Pros

-Outperforms better for simple highway structure which contains smaller number of nodes.

Cons

-Position information fully depends on formation of cells.

\subsection{Edge-Aware Epidemic Protocol (EAEP)}

EAEP [25] is a special type of protocol. The procedure of the protocol is that to broadcast messages among vehicles each vehicle piggybacks its own geographical position. After receiving a new rebroadcast message EAEP protocol take decision whether nodes will rebroadcast the message or not by using number of transmission from front and back nodes in a given time period. Although in EAEP a node does not know if it misses any messages

Pros

-Reduce control packet overhead by eliminating hello packets.

-Overcome simple flooding problem.

Cons

-Does not address the intermittent connectivity issue.

-High delay of data transmission.

\subsection{Distributed Vehicular Broadcast Protocol (DV-CAST)}

DV-CAST [8] is based on the connectivity as well connected, sparsely connected, and totally disconnected neighborhood that divides vehicles into three categories. For well connected neighborhood 
persistence scheme is used, for sparsely connected neighborhood vehicles can immediately rebroadcast with vehicles moving in the same direction after receiving the broadcast message, for totally disconnected neighborhood vehicles store the broadcast message until another vehicle enters into transmission range. When time is expired then discard the packets. In Distributed vehicular broadcast protocol to check whether the packet is redundant or not each vehicle uses a flag variable.

Pros

-By using flag variable check whether the packet is redundant or not.

Cons

-Control overhead is high.

-End to end data transfer delay is high.

\subsection{Secure Ring Broadcasting (SRB)}

In Secure Ring Broadcasting (SRB) [5] classifies nodes into three groups based on receiving power as Inner Nodes, Outer Nodes, and Secure Ring nodes. The nodes are close to sending node is consider Inner Nodes, far away from sending node is consider Outer Nodes and preferable distance from sending node is consider Secure Ring nodes.

\section{Pros}

-To get more stable routes minimize number of retransmission messages.

Cons

-Control packet overhead is high.

\subsection{Parameter less Broadcasting in Static to highly Mobile Wireless ad hoc(PBSM)}

In Parameter less broadcasting in static to highly mobile wireless ad Hoc (PBSM) [26] nodes does not need to know neighbor information. To eliminate redundant broadcasting it uses connected dominating sets (CDS) and neighbor elimination concepts. Two lists of neighboring vehicles: $\mathrm{R}$ and NR is maintained by each vehicle which helps to detect neighbors that already received and that which did not receive the packet. When timeout occur then rebroadcasts the packet.

Pros -Parameter less protocol does not consider vehicle position, direction and velocity.

Cons -Control Packet overhead is high.

\subsection{Preferred Group Broadcast(PGB)}

To prevent broadcast storm problem from route request broadcasting preferred group broadcast [27] is designed. In PGB from neighbor broadcasting each node can sense the level of signal strength. Rebroadcast message for only node with shortest timeout.

Pros -Reduce numbers of RREQ broadcasting

Cons - Not reliable broadcasting protocol.

\subsection{Urban Multihop Broadcast Protocol(UMB)}

UMB [28] is designed to solve collision and hidden node problems during message distribution in multi hop broadcast. In this protocol without any prior topology information sender node tries to select the furthest node in the broadcast direction for forwarding and acknowledging the packet. It performs well in higher packet loads \& vehicle traffic density.

\section{Pros}

-Overcome Packet collision and hidden node problems.

-Successfully performs at higher packet loads and vehicle traffic densities scenarios.

Cons -Waste Bandwidth

\subsection{Vector Based Tracing Detection(V-TRADE)}

Vector Based Tracing Detection [29] is a GPS based message broadcasting protocols which classifies the neighbors into different forwarding groups. To rebroadcast the message only a small subset of vehicles is selected for each group.

Pros

-Bandwidth utilization is improved.

Cons

-For selecting the next forwarding node in every hop routing overheads occur.

\subsection{Density-aware Reliable Broadcasting Protocol (DECA)}

DECA [30] does not require position knowledge and for broadcasting selects one neighbor which has the highest local density information. In the protocol received broadcast messages are included into periodic 
beacons thus a node can identify its neighbors, which have not received the messages and rebroadcast the messages for those neighbors.

Pros

-not require position knowledge

Cons

-Before timeout if all nodes don't identify anyone rebroadcast the message then they will rebroadcast the message.

\section{Geocast Based Routing Protocol}

Geocast routing is a location based multicast routing protocol which is used to send a message to all vehicles in a pre-defined geographical region. The selected area for transmission is called Zone of Relevance or ZOR. The main idea is that sender node need not to packet to nodes beyond the ZOR. Direct flooding strategy is used to reduce the amount of overhead and network congestion when packets are flooded. The various Geocast routing protocols are IVG, DG-CASTOR, DRG.

\subsection{Inter-Vehicle Geocast (IVG)}

IVG [31] is a Geocast routing protocol which is for disseminating safety messages to vehicles on highways. Timer based mechanism is used for forwarding message. For overcoming network fragmentation periodic broadcasts is used.

\subsection{Robust Vehicular Routing (ROVER)}

Robust Vehicular Routing [32] is a GeoCast based Routing Protocol which main goal is to send a message to all other vehicles within a specified Zone of Relevance (ZOR). In this protocol control packets are broadcasted in the network and the data packets are unicasted.

Pros

-Reliable geographical multicast protocol.

Cons

-High delay in data transfer because of redundant message.

-Control Packet overhead is high

-No of retransmission high

\subsection{Dynamic Time-Stable Geocast Routing (DTSG)}

Dynamic Time-Stable Geocast Routing [33] is mainly designed for sparse density networks. This protocol has two phases: Pre-stable phase helps the message to be disseminated within the region and stableperiod intermediate node uses store and forward method for a predefined time within the region

Pros

-Dynamically adjusts network density and the vehicles speed for better performance.

Cons

- No. of retransmission high

\section{Conclusion}

In this paper, we have investigated the pros and cons of different routing protocols that are applicable in vehicular communications for the development. The existing routing protocols for VANET are not efficient to meet every traffic scenarios. Thus design of an efficient routing protocol has taken significant attention. By studying different routing protocol in VANET we have seen that further performance evaluation is required to verify performance of a routing protocol with other routing protocols based on various traffic scenarios. The comparison of different VANET protocols features is essential to design a new proposal for VANET.

\section{Websites}

\section{References}

[1] Ericson, "Communication and Mobility by Cellular Advanced Radio", ComCar project, www.comcar.de,2002.

[2] Online, http://www.ist-drive.org/index2.html.

\section{Thesis Papers}

[3] W. Franz, H. Hartenstein, and M. Mauve, Eds., Inter-Vehicle-Communications Based on Ad Hoc Networking Principles-The Fleet Net Project. Karlshue, Germany: Universitatverlag Karlsuhe, November 2005.

[4] Forderer, D (2005). "Street-Topology Based Routing." Master's thesis, University of Mannheim, May 2005.

[5] Rainer Baumann, "Vehicular Ad hoc Networks", Master's Thesis in Computer Science, ETH Zurich (2004).

\section{Workshop Papers}

[6] Festag, et. al., "NoW-Network on Wheels: Project Objectives, Technology and Achievements", Proceedings of 6th International Workshop on Intelligent Transportations (WIT), Hamburg, Germany, March 2008.

[7] Lochert, C., Hartenstein, H., Tian, J., Fussler, H., Hermann, D., Mauve, M. (2003), "A routing strategy for vehicular ad hoc networks in city environments," Intelligent Vehicles Symposium, 2003. Proceedings. IEEE, vol., no., pp. 156-161, 9-11 June 2003. 
[8] O. K. Tonguz, N. Wisitpongphan, F. Bai, P. Mudalige and V. Sadekar, "Broadcasting in VANET", Proc. IEEE INFOCOM MOVE Workshop 2007, Anchorage, USA, (2007).

\section{Journal Papers}

[9] Perkins, C.; Belding-Royer, E.; Das, S. (July 2003)"Adhoc On-Demand Distance Vector (AODV) Routing".

[10] Johnson, D. B. and Maltz, D. A. (1996), "Dynamic Source Routing in Ad Hoc Wireless Networks," Mobile Computing, T. Imielinski and H. Korth, Eds., Ch. 5, Kluwer, 1996, pp. 153-81.

[11] Park, V.D., Corson, M.S. (1997), “A highly adaptive distributed routing algorithm for mobile wireless networks,” INFOCOM '97. Sixteenth Annual Joint Conference of the IEEE Computer and Communications Societies. Proceedings IEEE, vol.3, no., pp.14051413 vol.3, 7-12 Apr 1997.

[12] Karp, B. and Kung, H. T (2000), "GPSR: greedy perimeter stateless routing for wireless networks.” In Mobile Computing and Networking, pages 243-254, 2000.

[13] Lochert, C., Mauve, M., F“ussler, H., and Hartenstein, H., "Geographic routing in city scenarios," SIGMOBILE Mob. Comput. Commun. Rev., vol. 9, no. 1, pp. 69-72, 2005.

[14] R. A. Santos, "Performance evaluation of routing protocols in vehicular ad hoc networks," 2005.

Books

[15] Seet, B.-C., Liu, G., Lee, B.-S., Foh, C. H., Wong, K. J., Lee, K.-K. (2004), “A-STAR: A Mobile Ad Hoc Routing Strategy for Metropolis Vehicular Communications." NETWORKING 2004, 989-999.

\section{Symposium Paper}

[16] R. A. Santns, R. M. Edwards, A. Edwards and D. Belis, "A novel cluster-based location routing algorithm for intervehicular communication", Personal, Indoor and Mobile Radio Communications, IEEE proceedings of the 15th Annual Symposium, 2004.

\section{Proceedings Papers}

[17] Reichardt D., Miglietta M., Moretti L., Morsink P., and Schulz W., "CarTALK 2000 - safe and comfortable driving based upon inter-vehicle-communication," in Proc. IEEE IV'02.

[18] Morris R., Jannotti J., Kaashoek F., Li J., Decouto D., “CarNet: A scalable ad hoc wireless network system,” 9th ACM SIGOPS.

[19] Pei, G., Gerla, M., and Chen, T.-W. (2000), "Fisheye State Routing: A Routing Scheme for Ad Hoc Wireless Networks," Proc. ICC 2000, New Orleans, LA, June 2000.

[20] Naumov, V., Gross, T.R. (2007), "Connectivity-Aware Routing (CAR) in Vehicular Ad-hoc Networks," INFOCOM 2007. 26th IEEE International Conference on Computer Communications. IEEE, vol., no., pp.1919- 1927, 6-12 May, 2007.

[21] Yang Xia," Hierarchical Cluster Based Routing for Highly Mobile Heterogeneous MANET", 2007.

[22] Tao Song, Wei Xia, Tiecheng Song, Lianfeng Shen,"A Cluster-Based Directional Routing Protocol in VANET", International Conference on Communication and Mobile Computing, 2010.

[23] Yuyi Luo, Wei Zhang, Yangqing Hu,"A New Cluster Based Routing Protocol for VANET", IEEE Wireless Communications and Trusted Computing, 2010.

[24] M. Durresi, "Emergency broadcast protocol for intervehicle communications," 2005.

[25] M. Nekovee, B. BjamiBogason, "Reliable and efficient information dissemination in intermittently connected vehicular ad hoc networks", IEEE the 65th VTC'07 spring, Dublin, Ireland, 22-25 (2007).

[26] Adnan Afsar Khan, Ivan Stojmenovic, Nejib Zaguia, "Parameter less broadcasting in static to highly mobile wireless ad hoc, sensor and actuator networks", in Proc. ACM Int.Conference on Mobile Computing and Networking (MobiCom), Seattle, USA, (1999).

[27] V. Naumov, R. Baumann, and T. Gross, “An evaluation of inter-vehicle ad hoc networks based on realistic vehicular traces," Proc. ACM the 7th ACM MohiHoc'06, Florence, Italy, May 22-25 (2006).

[28] G. Korkmaz, "Urban multihop broadcast protocol for inter-vehicle communication systems".

[29] M. Sum, "GPS-based message broadcasting for Intervehicle", (2000).

[30] N. Na Nakom, and K. Rojviboonchai, "DECA: Density-Aware Reliable Broadcasting in Vehicular Ad-Hoc Networks," IEEE the 7th ECTICON20IO, Chiang Mai, Thailand, May 19-21 (2010).

[31] A. Bachir, "A Multicast Protocol", in Ad hoc Networks Inter-Vehicle Geocast. April 2003.

[32] M. Kihl,"Reliable Geographical Multicast Routing in Vehicular Adhoc Networks", 2007.

[33] Hamidreza Rahbar, Kshira sagar Naik, Amiya Nayak, "DTSG: Dynamic Time-Stable Geocast Routing in Vehicular Ad Hoc Networks", 2001.

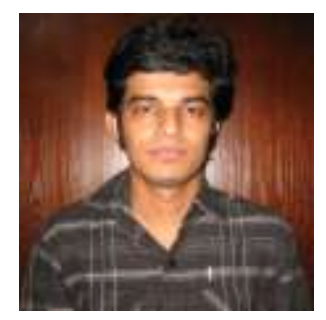

Bijan Paul received the BSc. (Hon's) and now he is an MSc student in the Department. of Computer Science \& Engineering, Shahjalal University of Science \& Technology, Bangladesh. His research interest includes VANET, Routing protocols, Wireless computing, and Human computer interaction.

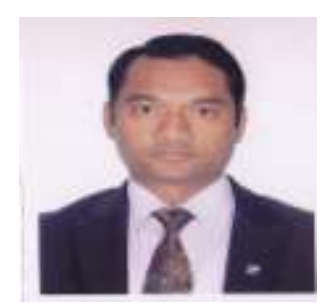

M.J. Islam received the BSc. (Hon's) and MSc. Degree in Electronics and Computer Science in 1995 and 1996 respectively from Shahjalal University of Science and Technology, Sylhet, Bangladesh. He received MASc in Electrical and Computer Engineering from Ryerson University, Toronto, ON, Canada, in 2003. In 2010, he received his PhD degree in Electrical Engineering from University of Windsor, Windsor, ON, Canada. Since 1997, he has been with the Computer Science and Engineering Department at Shahjalal University of Science and Technology, Sylhet, Bangladesh and promoted to Associate Professor in 2012. Mr. Islam has published 6 journal papers and 8 conference papers and received 3 best student paper awards. Mr. Islam is the Professional Engineer (PEng. PEO) in the province of Ontario, Canada. He is also IEEE member and the member of International Association of Engineers (IAENG). His research interests include but not limited to machine learning and computer vision, image processing, document image analysis and VANET. 